\title{
Morpho-functional state of the maxillofacial region in children with cerebral palsy
}

\author{
O. E. Idiev \\ Bukhara State medical Institute.
}

\section{Introduction}

The problem of cerebral palsy is one of the most urgent problems of medicine. The social significance of this problem is so great that it is quite natural that there is an increasing interest in it. In the available literature, there is no comprehensive data on the study of the frequency of dental anomalies among children with cerebral palsy, depending on the etiofactor, and on improving methods for their prevention and early treatment.

As is known from the literature, $70 \%$ of children with cerebral palsy have oral respiration.(Persin L. S. / / Orthodontics.-2003.-P. 94-106.) Oral respiration leads to a violation of the activity of the facial muscles, circular muscles of the mouth, tongue and to the development of dental anomalies (ZFA). Violations of myodynamic balance are observed between the buccal, masticatory, temporal and hyoid muscles. The myodynamic balance may be disturbed between the circular muscle of the mouth, the chin, and the muscles of the floor of the mouth. If the respiratory function is impaired, the activity of the circular mouth muscle increases several times compared to the norm, and its endurance is significantly reduced.

In the available literature, we were not able to find detailed information about the morphometric parameters of the head and THROAT of children with cerebral palsy and the relationship of these parameters with the functional state of the masticatory muscles. And as astrometrically parameters of FACE after the correction with dental correctors

The purpose of our study was to study the morphometric parameters of the head and maxillofacial region of children with cerebral palsy before and after the use of various dental correctors.

\section{Research problem:}

1)Study the anthropometric parameters of the head of children with cerebral palsy and compare them with the data of healthy children

2) Study the morphometric parameters of the maxillofacial region of children with cerebral palsy before correction with various dental correctors and compare them to the data of healthy children, taking into account gender

3) Study the functional state of the masticatory muscles in children with cerebral palsy, and compare them with the myographs of the masticatory muscles of healthy children.

4) compare the morphometric parameters of the maxillofacial region of children with cerebral palsy before and after correction with various dental correctors.

\section{Method of research:}

- Anthropometry

-teleradiography of the maxillofacial region

- panoramic radiography.

- electromyography of the masticatory muscles.

In accordance with the objectives of the study, patients were distributed by the etiological factor of the underlying disease, by the clinic of cerebral palsy, and by the period of bite formation (by age). 
In order to study the features of the structure of the dentition and its apical basis in prognatic bite, depending on the child's disease (cerebral palsy), we conducted an anthropometric study of diagnostic models of the jaws of the examined children. To clarify the parameters of prognatic bite in patients with cerebral palsy, we compared them with the data of the norm. The results of the analysis of the obtained data showed significant changes in the shape of the dentition in both the sagittal and transversal directions.

The size of the sagittal gap in the frontal part of the dentition was mainly in the range from 4.0 to 6.0 $\mathrm{mm}$, the sagittal gap of more than $6 \mathrm{~mm}$ met between the lateral teeth in the form of a two-sided displacement of molars by $1 / 2$ mound ( 1 degree) was $28.6 \%$, and the discrepancy of molars by the size of the mound ( 2 degree) was $32.4 \%$. Comparison of the true values of the transversal parameters of the dental arches in patients with cerebral palsy with the norm showed that the measured diagnostic models showed that the anterior width of both the upper and lower dentition was less than the norm. The posterior width of the dental arches of the lower jaw was significantly greater by $1.20 \mathrm{~mm}(\mathrm{~T}=2.2)$ in sick children compared to the norm, while there was no statistically significant difference in the posterior width of the upper dentition. The study of the width and length of the apical base of the dentition revealed that they were both on the upper and lower jaw significantly larger in the examined children compared to the norm, with the exception of the main group was similar to the norm parameter.

When comparing the anthropometric indicators of prognatic bite in children who developed during pregnancy or have a history of other adverse factors of the underlying disease, we found some deviations in the frequency and severity of the above symptoms between the selected groups. In order to study the features of the structure of the dentition and its apical basis in prognatic bite, depending on the child's disease (cerebral palsy), we conducted an anthropometric study of diagnostic models of the jaws of the examined children. To clarify the parameters of prognatic bite in patients with cerebral palsy, we compared them with the data of the norm. The results of the analysis of the obtained data showed significant changes in the shape of the dentition in both the sagittal and transversal directions.

The size of the sagittal gap in the frontal part of the dentition was mainly in the range from 4.0 to 6.0 $\mathrm{mm}$, the sagittal gap of more than $6 \mathrm{~mm}$ met between the lateral teeth in the form of a two-sided displacement of molars by $1 / 2$ mound ( 1 degree) was $28.6 \%$, and the discrepancy of molars by the size of the mound ( 2 degree) was $32.4 \%$. Comparison of the true values of the transversal parameters of the dental arches in patients with cerebral palsy with the norm showed that the measured diagnostic models showed that the anterior width of both the upper and lower dentition was less than the norm. The posterior width of the dental arches of the lower jaw was significantly greater by $1.20 \mathrm{~mm}(\mathrm{~T}=2.2)$ in sick children compared to the norm, while there was no statistically significant difference in the posterior width of the upper dentition. The study of the width and length of the apical base of the dentition revealed that they were both on the upper and lower jaw significantly larger in the examined children compared to the norm, with the exception of the main group was similar to the norm parameter.

When analyzing the parameters of prognathic malocclusion in children with various degree of mental development is set statically significant difference in the amount of mesiodistally sizes 12 teeth, rear width of the dentition and length of apical base. In the upper jaw, this discrepancy was statistically significant in the second group of children (with mental retardation) compared to the first group (with normal intelligence). At the same time, the most statistically significant difference was established in relation to the bilateral mismatch of the lateral teeth by the size of the tubercles, where the number of such children in the second group was $18.6 \%$ more $(\mathrm{T}=2.4)$ than in the first group. The remaining symptoms in the second group also differed both in severity and in the frequency of their combination with each other.

Studying the features of functional changes in masticatory and mimic muscles using electromyography allowed us to obtain an indirect characteristic of the mechanism of development of dental anomalies in the examined patients and develop special gymnastic exercises for pathogenetic therapy of dental anomalies and 
correction of violations of the peripheral sound-producing side of speech.

In patients with hyperginetic cerebral palsy, these disorders were characterized by asymmetry between the right and left masticatory muscles, as a result of hyperkinesis of these muscles. These significant uneven changes in the tonic bioelectric activity of the muscles contributed to excessive training of their individual groups (in particular, facial muscles, tongue muscles) and the weakness of others

(chewing, soft palate muscles), which can complicate orthodontic treatment or cause relapses.

Electromyograms obtained in patients with cerebral palsy during compression of the dentition and during chewing showed a decrease in the bioelectric activity of the masticatory muscles compared to the norm, especially in children suffering from hyperkinetic form of the disease.

In order to further study the functional state of the masticatory muscles, we determined the degree of involvement of individual muscles during function, i.e., the coordination between the contraction of the bilateral pair of proper masticatory and temporal muscles. The use of a statistical method for determining the correlation between functioning masticatory muscles showed a low or medium degree of correlation, but the correlation coefficient between the features was not the same, i.e. the correlation relationship was uneven. The above suggests a violation of the coordination of the function of the bilateral pairs of masticatory muscles in patients with cerebral palsy, in contrast to the control group.

Basic principles and features of providing orthodontic care to patients with PD in the process of their General medical examination.

A high prevalence of morphological and functional disorders dentoalveolar system in patients with PDD, identified in later stages of the disease, and the establishment of the fact of their increase with age was the basis for the development of proposals relating to organizacii and methods of orthodontic care to such patients in terms of their General examination.

For patients with cerebral palsy, the organization of speech therapy sessions in combination with orthodontic therapy, in order to correct the peripheral speech apparatus, is pathogenetically justified. Currently, speech therapists are most often engaged in speech disorders in such patients, while this task cannot be assigned only to speech therapists, since most of these children need the help of an orthodontist. The orthodontist must not only correct the bite, but also overcome bad habits. This work should begin as early as possible, in order to prevent the formation of the usual pathological compensatory stereotype.

The role of an orthodontist in the complex treatment of patients with cerebral palsy is also that he, in collaboration with neuropsychiatrists, speech therapists and physical therapy specialists, provides systematic, systematic, dynamic monitoring of the state of the dental system that affects the patient's speech-motor abilities, thereby participating in this complex correctional care. Due to this complex treatment, some success can be achieved, especially if treatment begins early. However, dentists are still not sufficiently familiar with the clinical manifestations of cerebral palsy and methods of providing dental care, including orthodontic, to these patients, which does not remain without influence on the prognosis of treatment of this complex brain pathology. The number of children in need of orthodontic care increases in parallel with the age of the child, so in the period of milk bite, the need for active treatment of these patients is $23.9+-4.7 \%$. This is significantly lower by $39.7 \%$ compared to children in the period of permanent bite. The network of such orthodontic offices and centers should be included in the number of pre-school dental sections headed by a district children's dentist.

It should be noted that in the orthodontic treatment of children with spasticity or lethargy of large muscle groups (including articulation), hyperkinesis of the head, lower jaw, muscles, face and tongue with constant salivation, impaired mental development and emotional instability, the existing load standards of orthodontists (and other dentists) are overstated. Based on this, we consider it appropriate to review the staffing of dental, including orthodontic, offices serving patients with cerebral palsy. 


\section{CONCLUSIONS}

This is especially noticeable in children suffering from a hyperkinetic form of the disease, where hyperkinesis of the muscles of the articulatory system often contributes, where hyperkinesis of the muscles of the articulatory system often contributes to increased erasability of hard tissues of the teeth, a decrease in the height of the bite, mixing of the lower jaw and the establishment of incorrect habitual occlusion. On the other hand, morphological abnormalities in the dental apparatus often found in these children also contribute to the emergence and consolidation of bad habits. Knowledge of these features is important for orthodontists, and overcoming them will increase the effectiveness of orthodontic treatment and speech therapy training for this contingent of children.

\section{References:}

1. Afanasyeva L. R. Dental anomalies and their relationship with respiratory diseases in children with intellectual disabilities. Proceedings of young scientists: Collection of scientific papers. Minsk. 2001. C. 324-327.

2. Baizhanov B, B. Dental anomalies in patients with cerebral palsy and features of orthodontic care in terms of their complex treatment: Diss.... Candidate of medical Sciences. Tashkent $1982 .$.

3. Badalyan L. O., Zhurba L. T., Vsevolzhskaya N. M. Guide to neurology of childhood.- Kiev: Zdorov'ya, 1980. $-528 \mathrm{p}$.

4. Bogatov A. I. Our method of treatment of periodontal diseases in children with cerebral palsy. // Samar. med. Journal. 2001. N 4. C. 44

5. Danilevsky N. F., Vyshniak G. N., A. M. politun Periodontics children.- Kiev: Zdorovye, 1981. - 296s.

6. Distel V. A. et al. Dentofacial anomalies and deformations: the Main reasons for the development. Moscow. N. Novgorod. Med. kN. Izd-vo ngma. 2001. C. 102 p.

7. Korchagina V. V. State of the oral cavity in children suffering from cerebral palsy, spinal hernias and myopathies. Autoref. dis. on the map. scientist. step. candidate of medical Sciences. Moscow 1995. C. 21

8. Korchagina V. V., Dyakova S. V., Liljin E. T. Dentistry 1996; 6: 39-44.

9. Korchagina V. V. State of the oral cavity in children with defects of the Central nervous system and musculoskeletal system of congenital and hereditary nature [cerebral palsy, spinal hernias, myopathies].//Stomatology. 1996. 75. no. 6. C. 39-44.

10. Ogonyan V. R. Dental status of children with cerebral palsy SB. St. Volgograd. 1996. C. 15-20.

11. Okushko V. P. Dental anomalies associated with bad habits and their treatment. Autoref. dis. on the map. scientist. step. Cand. med. sciences'.Moscow. 1965. C. 20

12. Oleynik E. A. Diagnostics of dental and periodontal diseases in children with organic damage to the Central nervous system. Autoref. dis. on the map. scientist. step. Cand. med. sciences'. Voronezh. 2000. C. 20 